\title{
A SHARP CONDITION FOR UNIVALENCE IN EUCLIDEAN SPACES
}

\author{
JULIAN GEVIRTZ
}

\begin{abstract}
Let $B \subset E^{k}$ be a ball. It is shown that if $f: B \rightarrow E^{k}$ is a local homeomorphism for which the infinitesimal change in length is bounded above by $M$ and for which the infinitesimal change in volume is bounded below by $m^{k}$, where $M / m \leqslant 2^{1 / k}$, then $f$ is univalent. This result is numerically sharp.
\end{abstract}

Throughout, $G$ will be an open set in $E^{k}$, the $k$-dimensional Euclidean space, $f: G \rightarrow E^{k}$ will be a local homeomorphism and $0<m<M$ will be real numbers. We denote by $Q(G, m, M)$ the set of all $f$ which satisfy the following two conditions for all $x \in G$ :

$$
\begin{gathered}
M \geqslant \limsup _{y \rightarrow x} \frac{\|f(y)-f(x)\|}{\|y-x\|}, \\
m \leqslant \liminf _{y \rightarrow x} \frac{\|f(y)-f(x)\|}{\|y-x\|} .
\end{gathered}
$$

John [2], [3] calls such $f$ quasi-isometric. As a slight improvement of a result of John [3], the author [1] showed that if $M / m \leqslant(4+2 \pi) /(4+\pi)$ $=1.439 \cdots$, then all $f$ in $Q(B, m, M)$ are univalent, where $B$ is a ball. It seems reasonable to conjecture that this constant can be replaced by 2 , but this appears difficult. Here, instead of approaching this conjecture by trying to improve the result of [3] still further, we shall consider a different class of mappings for which it is possible to prove a numerically sharp theorem. The result we get would follow if the conjecture were established. Our argument is based on the standard proof of the isoperimetric inequality $L^{2} \geqslant 4 \pi A$ on minimal surfaces of least area.

We define $Q^{\prime}(G, m, M)$ to be the set of all $f$ for which (1) together with

$$
m^{k} \leqslant \liminf _{r \rightarrow 0} \frac{\mu(f(B(x, r)))}{\mu(B(x, r))}
$$

hold for all $x \in G$, where $\mu$ denotes Lebesgue measure and $B(x, r)$ is the ball of radius $r$ and center $x$. Our result is the following

THEOREM. If $f \in Q^{\prime}(B(x, r), m, M)$ and $M / m \leqslant 2^{1 / k}$, then $f$ is univalent. Furthermore, $2^{1 / k}$ cannot be replaced by any larger constant.

Received by the editors July $16,1975$.

AMS (MOS) subject classifications (1970). Primary 26A57, 53A99.

Key words and phrases. Univalent, local homeomorphism, quasi-isometric mapping.

O American Mathematical Society 1976 
Before proving this theorem we discuss the relationship between $Q^{\prime}(G, m, M)$ and $Q(G, m, M)$. Obviously, $Q(G, m, M) \subset Q^{\prime}(G, m, M)$. We show that

$$
Q^{\prime}(G, m, M) \subset Q\left(G, m^{k} / M^{k-1}, M\right) .
$$

The obvious Vitali covering argument shows that if $f \in Q^{\prime}(G, m, M)$ is a homeomorphism on $G$, then $\mu(f(G)) \geqslant m^{k} \mu(G)$. If $X \subset E^{k}, B(X, \varepsilon)$ will be the open $\varepsilon$-neighborhood of $X$. For $a, b \in E^{k},[a, b]$ will denote the line segment connecting $a$ and $b$. Furthermore, $s_{k}=\mu(B(x, 1))$. We need the following fact proved by John [2]:

Lemma 1. If $h: G \rightarrow E^{k}$ satisfies (1) for all $x \in G$ and if $[a, b] \subset G$, then $\|h(b)-h(a)\| \leqslant M\|b-a\|$.

We now prove (3). We must show that for $f \in Q^{\prime}(G, m, M)$ the lim inf in (2) is bounded below by $m^{k} / M^{k-1}$ for all $x \in G$. This being a local property, we may assume that $f$ is one-to-one in $G$. Let $r>0$ be such that $B(f(x), r)$ $\subset f(G)$. Let $\quad y \in J=f^{-1}(B(f(x), r / 2))$ and $0<\varepsilon<r / 2$. Let $T(\varepsilon)$ $=B([f(x), f(y)], \varepsilon)$. If $z \notin f^{-1}(T(\varepsilon))$, then Lemma 1 implies that $z$ must be at least $\varepsilon / M$ from $f^{-1}([f(x), f(y)])$ which is a curve joining $x$ and $y$. Thus $f^{-1}(T(\varepsilon))$ contains an $(\varepsilon / M)$-neighborhood of this curve. Hence $\mu\left(f^{-1}(T(\varepsilon))\right)$ is bounded below by $\|x-y\| s_{k-1}(\varepsilon / M)^{k-1}$. But $\mu(T(\varepsilon))$ is given by

$$
\|f(x)-f(y)\| s_{k-1} \varepsilon^{k-1}+s_{k} \varepsilon^{k} .
$$

Since $\mu(T(\varepsilon)) \geqslant m^{k} \mu\left(f^{-1}(T(\varepsilon))\right)$, we have

$$
\|f(x)-f(y)\| s_{k-1} \varepsilon^{k-1}+s_{k} \varepsilon^{k} \geqslant m^{k}\|x-y\| s_{k-1}(\varepsilon / M)^{k-1} .
$$

Allowing $\varepsilon \rightarrow 0$, we see that $\|f(x)-f(y)\|$ is bounded below by $\left(m^{k} / M^{k-1}\right)$ - $\|x-y\|$, which gives (3) since $y \in J$ is arbitrary.

We note in passing that the $m^{k} / M^{k-1}$ appearing in (3) cannot be replaced by any larger number as is shown by the linear transformation whose matrix with respect to some orthonormal basis of $E^{k}$ is diagonal with entries $m^{k} / M^{k-1}, M \cdots, M$.

Before beginning the proof of our theorem we need some terminology and facts. In what follows we shall not distinguish explicitly between a curve or surface as such and the set of points that lie on it. This should cause no confusion. Arc length and plane measure will be denoted by $\lambda$ and $\mu_{2}$, respectively. For brevity we write $B(r)=B(0, r)$ and denote closed balls with a bar. Also, $D=\bar{B}(1)$ in $E^{2}$. For a closed curve $C$ in $E^{k}$ we say that the surface given by $\psi: D \rightarrow E^{k}$ spans $C$ if $\left.\psi\right|_{\partial D}$ is a parametrization of $C$ and we say that $C$ links with $A \subset E^{k}$ if $C \cap A=\varnothing$ and every surface which spans $C$ intersects $A$.

Lemma 2. If $h: G \rightarrow E^{k}$ satisfies (1) in $G$ and if $C$ is a rectifiable curve lying in $G$, then $\lambda(h(C)) \leqslant M \lambda(C)$.

This is an obvious consequence of Lemma 1.

Lemma 3. Let $k \geqslant 3$. Let $B$ be an open ball in $E^{k}$ and let $h: \bar{B} \rightarrow E^{k}$ be a 
continuous mapping for which $\left.h\right|_{B}$ is a homeomorphism. Let $h(a)=h(b)$, where $a \neq b$ and $a, b \in \partial B$. Let $C^{\prime}$ and $C^{\prime \prime}$ be two simple curves which connect $a$ and $b$ in $B$ and on $\partial B$, respectively. Let $A \subset B$ be compact. If $C^{\prime} \cup C^{\prime \prime}$ links with $A$, then $h\left(C^{\prime}\right)$ links with $h(A)$.

This lemma is intuitively clear and a simple proof may be found in [1].

Let $H \subset E^{k}, k \geqslant 3$, be any closed half plane. Let $x=\left(x_{1}, \ldots, x_{k}\right)$ be a Cartesian coordinate system for which $H=\left\{x: x_{2} \geqslant 0\right.$ and $x_{j}=0$ for $j$ $\geqslant 3\}$. We define $W_{H}: E^{k} \rightarrow H$ by $W_{H}(x)=\left(x_{1},\left(x_{2}^{2}+\cdots+x_{k}^{2}\right)^{1 / 2}, 0, \ldots, 0\right)$.

Lemma 4. Let $f \in Q^{\prime}(G, m, M)$ be one-to-one in $G$ and let the closure of $X$ be contained in $G$. Assume that $f(X)$ is an open subset of some plane $P$ lying in $E^{k}$. Then $\mu_{2}(f(X))$ is bounded below by $\left(\mathrm{m}^{k} / M^{k-2}\right) \mu_{2}\left(W_{H}(X)\right)$.

Proof. First of all, we can assume that $W_{H}(X)$ lies at a positive distance from $L$, the edge of $H$, since obviously $\mu_{2}\left(W_{H}(X-\bar{B}(L, \varepsilon))\right)$ $\rightarrow \mu_{2}\left(W_{H}(X)\right)$ as $\varepsilon \rightarrow 0$. Secondly, since the inverse of a homeomorphism $h$ in $Q(G, m, M)$ is in $Q(h(G), 1 / M, 1 / m)$, we see by (3) that

$$
f^{-1} \in Q\left(f(G), 1 / M, M^{k-1} / m^{k}\right) .
$$

Also, $W_{H}$ is Lipschitz continuous with constant 1 . These two facts imply that if $A \subset P$ and $\mu_{2}(A)=0$, then $\mu_{2}\left(W_{H}\left(f^{-1}(A)\right)\right)=0$. Since $f(X)=A \cup R$, where $\mu_{2}(A)=0$ and $R$ is a countable disjoint union of squares, we may assume that $f(X)$ is itself a square. Our first assumption yields

$$
\mu(B(X, \varepsilon)) \geqslant s_{k-2} \varepsilon^{k-2} \mu_{2}\left(W_{H}(X)\right)-o\left(\varepsilon^{k-2}\right) .
$$

However, for $\varepsilon$ small $f(B(X, \varepsilon))$ is contained in $B(f(X), M \varepsilon)$ whose measure is $s_{k-2}(M \varepsilon)^{k-2} \mu_{2}(f(X))$ to within $o\left(\varepsilon^{k-2}\right)$ by our other assumption. By the fact mentioned just after (3),

$$
s_{k-2}(M \varepsilon)^{k-2} \mu_{2}(f(X)) \geqslant m^{k} s_{k-2} \varepsilon^{k-2} \mu_{2}\left(W_{H}(X)\right)-o\left(\varepsilon^{k-2}\right) .
$$

Upon dividing by $s_{k-2} \varepsilon^{k-2}$ and letting $\varepsilon \rightarrow 0$ we obtain our result.

We now begin the proof of the theorem. Clearly we may assume that $x=0$. We suppose that $f \in Q^{\prime}(B(r), m, M)$ is not univalent and show that this implies that $M / m>2^{1 / k}$. First we do the case $k \geqslant 3$. The case $k=2$ may be handled with minor modifications or may be deduced from the higher dimensional case as we show later. Let $r_{0}=\inf \{s: f$ is not univalent in $B(s)\}$. Then $r>r_{0}>0$ and there are two distinct points $a$ and $b$ on $\partial B\left(r_{0}\right)$ for which $f(a)=f(b)$. We have $r_{0}>0$, because the mapping is a local homeomorphism. The existence of the two points can be justified as follows: There exist two sequences $\left\{a_{n}\right\}$ and $\left\{b_{n}\right\}$, which can be assumed convergent, such that $a_{n} \neq b_{n}$, $f\left(a_{n}\right)=f\left(b_{n}\right)$ and $a_{n}, b_{n} \in B\left(r_{0}+1 / n\right)$. If $a$ and $b$ are the limits of these sequences, then $a \neq b$, since $f$ is a local homeomorphism. Also, we have $a, b \in \bar{B}\left(r_{0}\right)$ and the definition of $r_{0}$ implies that $a, b \in \partial B\left(r_{0}\right)$.

Let $P$ be the plane containing $a, b$ and 0 . Let $L$ be the line in $P$ through 0 parallel to $[a, b]$ and let $H \subset P$ be the closed half plane with edge $L$ containing $a$ and $b$. Let $c$ be the midpoint of $[a, b]$ and let $K=B(c,\|a-b\| / 2)$ $\cap(H-L) \cap B\left(r_{0}\right)$. Let $C=\partial K$ and let $C^{\prime}$ denote the part of $C$ which connects $a$ and $b$ inside $B\left(r_{0}\right)$. Simple geometric considerations show that 


$$
\left(\lambda\left(C^{\prime}\right)\right)^{2}<2 \pi \mu_{2}(K)
$$

Since $f(a)=f(b), f\left(C^{\prime}\right)$ is a closed curve. Evidently, for any $\varepsilon>0$ there are $n=n(\varepsilon)$ consecutive points $f(a)=p_{0}, p_{1}, \ldots, p_{n}=f(a)$ on $f\left(C^{\prime}\right)$ such that the piece of $f\left(C^{\prime}\right)$ between $p_{i-1}$ and $p_{i}$ is contained in $B\left(p_{i}, \varepsilon\right)$, for $1 \leqslant i \leqslant n$. Let $C(\varepsilon)$ denote the closed piecewise linear curve formed by connecting these $n$ points by line segments in order.

For $x \in H$, let $S(x)$ be the $(k-2)$-dimensional sphere $W_{H}^{-1}(x)$. If $x \in K$, then $C$ links with $S(x)$. Were this not the case, there would be a surface spanning $C$ but disjoint from $S(x)$. Applying $W_{H}$ to this surface we get a surface spanning $C$ and lying in $H$ but not containing $x$. This, however, is impossible since $x \in K$, the interior of $C$. Thus Lemma 3 implies that for $x \in K, f\left(C^{\prime}\right)$ links with $f(S(x))$. There is a $\delta=\delta(\varepsilon)>0$, which goes to 0 when $\varepsilon$ goes to 0 , such that $C(\varepsilon)$ links with $f(S(x))$ for each $x \in K$ whose distance from $C$ is at least $\delta$. This follows since if one continuously deforms a closed curve which links with a set $A$ without touching $A$, then the resulting curve links with $A$.

For $p \in E^{k}$ we denote by $d(\varepsilon, p)$ the surface spanning $C(\varepsilon)$ generated by the line segments $[p, x], x \in C(\varepsilon)$. This surface is a union of triangles. We denote by $\tau(p)$ the sum of the angles $p_{i-1} p p_{i}, 1 \leqslant i \leqslant n$. Here angles lie between 0 and $\pi$. The function $\tau$ is well defined and continuous for $p \neq p_{0}, p_{1}, \ldots, p_{n}$ and approaches 0 as $\|p\| \rightarrow \infty$. Let $\psi(p)$ be the same sum except that $2 \leqslant i \leqslant n-1$. Clearly, $\psi$ is even continuous at $p_{0}$. If $\psi(f(a)) \leqslant 2 \pi$, then we can "lay the surface $d(\varepsilon, f(a))$ out" in $E^{2}$ by placing the triangle $p_{0} p_{1} p_{2}$ in $E^{2}$, then placing $p_{0} p_{2} p_{3}$ next to it so that the sides $\left[p_{0}, p_{2}\right]$ of both triangles coincide, etc. In this case we set $q=f(a)$. If, on the contrary, $\psi(f(a))>2 \pi$, then the facts that $\tau(p) \geqslant \psi(p), \psi(p) \rightarrow \psi(f(a))>2 \pi$ as $p \rightarrow f(a)$ and $\tau(p)$ $\rightarrow 0$ as $\|p\| \rightarrow \infty$ imply, by the intermediate value theorem, that there is a $q \notin C(\varepsilon)$ for which $\tau(q)=2 \pi$. In this case, the surface $d(\varepsilon, q)$ can be "laid out" in $E^{2}$ by placing the triangles $p_{i-1} q p_{i}$ so that the sides $\left[p_{i}\right.$, $q$ ] of $p_{i-1} q p_{i}$ and $p_{i} q p_{i+1}$ coincide, for $1 \leqslant i \leqslant n$, where $p_{n+1}=p_{1}$.

In either case the "image" of $d(\varepsilon, q)$ is a polygon $T$ and $\partial T$ is the "image" of a part of $C(\varepsilon)$. (If $\psi(f(a)) \neq 2 \pi$ and there are no degenerate triangles, then $\partial T$ will be the "image" of all of $C(\varepsilon)$.) Moreover, the area of $T$ is the sum of the areas of the triangles making up $d(\varepsilon, q)$. If we now apply Lemma 4 to each of these triangles, we see that $\mu_{2}(T)$ is bounded below by $\left(m^{k} / M^{k-2}\right)$ $\cdot\left(\mu_{2}(K)-(\delta)\right)$, since the image under $W_{H}$ of the set $f^{-1}\left(f\left(B\left(r_{0}\right)\right) \cap d(\varepsilon, q)\right)$ contains all the points of $K$ whose distance from $C$ is at least $\delta$. The usual isoperimetric inequality gives us that $(\lambda(\partial T))^{2} \geqslant 4 \pi \mu_{2}(T)$. But

$$
\lambda(\partial T) \leqslant \sum_{i=1}^{n}\left\|p_{i}-p_{i-1}\right\| \leqslant \lambda\left(f\left(C^{\prime}\right)\right) \leqslant M \lambda\left(C^{\prime}\right),
$$

the last inequality following from Lemma 2. Thus $M^{2}\left(\lambda\left(C^{\prime}\right)\right)^{2}$ $\geqslant 4 \pi\left(m^{k} / M^{k-2}\right) \mu_{2}(K)-O(\delta)$. Allowing $\varepsilon$ to go to 0 and substituting (4), we obtain the desired inequality.

We now discuss the two dimensional case. For $k \geqslant 2$ we view $E^{k}$ as $E^{2} \times E^{k-2}$. Let $U \subset E^{2}$ and $V \subset E^{k-2}$ and let $g: U \rightarrow E^{2}$ and $h: V$ $\rightarrow E^{k-2}$. Define the mapping $g \times h$ by $g \times h(x, y)=(g(x), h(y))$ for $(x, y)$ $\in U \times V$. Let $B$ be an open disc in $E^{2}$ and $f \in Q^{\prime}(B, m, M)$. Let $h(y)=M y$ 
for $y \in E^{k-2}$. It is clear that $f \times h \in Q^{\prime}\left(B \times E^{k-2}, m^{\prime}, M\right)$, where $m^{\prime}$ $=\left(m^{2} M^{k-2}\right)^{1 / k}$. For $k \geqslant 3$, by what we have already proved $f \times h$ will be univalent in any ball contained in $B \times E^{k-2}$ if $M / m^{\prime} \leqslant 2^{1 / k}$, that is, if $M / m \leqslant \sqrt{2}$. Since $f$ is univalent in $B$ if $f \times h$ is univalent in some set containing $B \times\{0\}$, we obtain the result for $k=2$ once we have it in the higher dimensional case.

Finally, we show that the result is numerically sharp. Let $E^{2}$ be given polar coordinates $(r, \theta)$. Let $U$ be the right half plane, $-\pi / 2<\theta<\pi / 2$. Define $g(r, \theta)=(r,(2+\beta) \theta)$. If $h$ is as in the preceding paragraph with $M=2+\beta$, we see that $g \times h \in Q^{\prime}\left(U \times E^{k-2}, m, 2+\beta\right)$, where $m^{k}=(2+\beta)^{k-1}$. For $\beta>0, g \times h$ is not one to one in the $k$-dimensional ball $B(x, 1)$, where $x=(1,0, \ldots, 0)$. Since $M / m \rightarrow 2^{1 / k}$ as $\beta \rightarrow 0$, the proof is complete.

\section{REFERENCES}

1. J. Gevirtz, On the univalence of quasi-isometric mappings, Rev. Colombiana Mat. 7 (1973), 125-132.

2. F. John, On quasi-isometric mappings. I, Comm. Pure Appl. Math. 21 (1968), 77-110. MR 36 \# 5716.

3. $39 \# 6055$.

Instituto de Matematica, Universidad Católica de Chile, Casilla 114-D, Santiago, CHILE

Current address: Department of Mathematics, University of Alabama, Birmingham, Alabama 35294 